\title{
CHEBYCHEV APPROXIMATION IN LOCALLY COMPACT SPACES
}

\author{
JOSEPH BRAM
}

It is well known that if $f$ is a real continuous function on the unit interval I, then the best polynomial $p$ of degree $m$, in the Chebychev sense, that approximates $f$ has the property that if $e$ is the error function, $e=f-p$, then there exist $m+2$ points $x_{0}<x_{1}<\cdots<x_{m+1}$ in $I$ such that $(-1)^{i} e\left(x_{i}\right)=u\|e\|$, where $u= \pm 1$, and

$$
\|e\|=\max \{|e(x)|: x \in I\} .
$$

Moreover, this $p$ is unique, and is characterized by this property.

In this paper we shall give a generalization of this result (except for uniqueness, which does not hold) on a locally compact space $X$.

Let $B=C(X)$ be the Banach space of all continuous real functions on $X$, vanishing at infinity, with $\|f\|=\max \{|f(x)|: x \in X\}$. Let $g_{1}, \cdots, g_{n}$ be $n$ fixed functions in $B$, linearly independent, and let $P=\mathrm{V}\left\{g_{1}, \cdots, g_{n}\right\}$. The Chebychev approximation problem is to find $p_{0}$ in $P$ such that $\left\|f-p_{0}\right\| \leqq\|f-p\|$ for all $p$ in $P$. We can then assert:

Theorem. Let $f \in B, p_{0} \in P, e_{0}=f-p_{0}$. Then n.a.s.c. that $\left\|f-p_{0}\right\|$ $\leqq\|f-p\|$ for all $p$ in $P$ is that for some $r \leqq n$, there exist $r+1$ points $x_{0}, \cdots, x_{r}$ in $X$ such that the $n \times(r+1)$ matrix $\left\{g_{i}\left(x_{j}\right)\right\}, i=1,2, \cdots, n$; $j=0,1, \cdots, r$ has rank $r$, and such that if the first $r$ rows are independent (renumber the $g_{i}$ if necessary), and $a_{i}$ is the $\operatorname{sgn}$ of the cofactor of $a_{i}$ in the matrix

$$
\left(\begin{array}{ccc}
a_{0} & \cdots & a_{r} \\
g_{1}\left(x_{0}\right) & \cdots & g_{1}\left(x_{r}\right) \\
\vdots & & \vdots \\
g_{r}\left(x_{0}\right) & \cdots & g_{r}\left(x_{r}\right)
\end{array}\right)
$$

then $e_{0}\left(x_{j}\right)=a_{j}\left\|e_{0}\right\| u$ for all $j$ such that $a_{j} \neq 0$, where $u=1$ or $u=-1$.

In general, $p_{0}$ is not unique. Also for a given $p_{0}$ satisfying the conditions of the theorem, the points $x_{0}, \cdots, x_{r}$ are not unique, and not even the integer $r$ is unique.

Finally, we remark that there is at least one important special case where the integer $r$ of the theorem can have only the value

Presented to the Society, October 26, 1957; received by the editors June 3, 1957. 
$r=n$. This is the case where $X$ is a compact subset of the real line and $g_{i}(x)=x^{i-1}, i=1,2, \cdots, n$. It is this case that gives the classical theorem stated at the beginning (except for uniqueness, which must be proved separately).

To prove our theorem, we go to the dual space $B^{*}$ of $B$. We know that $B^{*}$ is the space of all regular finite signed measures on $X$; the measurable sets are the Borel sets of $X$. For every $F$ in $B^{*}$, we have the Jordan decomposition $F=F_{1}-F_{2}$, where $F_{1}$ and $F_{2}$ are (positive) measures on disjoint subsets. If we put $G=F_{1}+F_{2}$, then $\|F\|=\int_{X} d G$.

Throughout the following, we shall let $N=\left\{F \in B^{*}: \int p d F=0\right.$ for all $p$ in $P\}$, and $S$ denotes the unit sphere of $B^{*}$. We have $(B / P)^{*} \cong N$, an algebraic isomorphism and an isometry, so that inf $\{\|f-p\|: p \in P\}$ $=\|f+P \mid\|=\sup \left\{\int f d F: F \in N \cap S\right\}$, where $\||f+P \|| \mid$ is the norm in $B / P$.

Since $P$ is finite dimensional, there is certainly some $p_{0}$ in $P$ such that $\left\|f-p_{0}\right\|=\inf \{\|f-p\|: p \in P\}$. We let $e_{0}=f-p_{0}$. Since $N \cap S$ is $W^{*}$-compact, there is some $F_{0}$ in $N \cap S$ such that $\int f d F_{0}=\sup \left\{\int f d F\right.$ : $F \in N \cap S\}$. Then $\int e_{0} d F_{0}=\int\left(f-p_{0}\right) d F_{0}=\int f d F_{0}=\left\|f-p_{0}\right\|=\left\|e_{0}\right\|$. We let $C=\left\{F \in N \cap S: \int e_{0} d F=\left\|e_{0}\right\|\right\}$. Then $C \neq 0$. Since $C$ is convex and $W^{*}$-compact, $C$ is generated by its extreme points.

For every $F$ in $B^{*}$, we let $\Lambda(F)$ denote the support of $F$.

Lemma 1. Let $F_{0}$ be an extreme point of $C$. Let $P_{0}$ denote the space of functions of $P$, restricted to $\Lambda\left(F_{0}\right)$, and let $m$ be the dimension of $P_{0}$, so $m \leqq n$. Then $\Lambda\left(F_{0}\right)$ contains exactly $m+1$ points. Moreover, $\left|e_{0}(x)\right|$ $=\left\|e_{0}\right\|$ for every $x$ in $\Lambda\left(F_{0}\right)$.

Proof. The last assertion follows immediately from the facts that $\int e_{0} d F_{0}=\left\|e_{0}\right\|$ and $\left\|F_{0}\right\|=1$.

Let $F_{0}=F_{1}-F_{2}$ denote the Jordan decomposition of $F_{0}$, and set $G=F_{1}+F_{2}$. Let $v=e_{0} /\left\|e_{0}\right\|$. Then $v(x)=1$ on $\Lambda\left(F_{1}\right), v(x)=-1$ on $\Lambda\left(F_{2}\right)$, and $\int g d F_{0}=\int g v d G$ for every $g$ in $B$.

Let $H=L_{2}(G)$. Then $P_{0}$ has dimension $m$ in $H$, and $\int p d F_{0}=\int p v d G$ $=0$ for all $p$ in $P_{0}$, so that $H$ is at least $m+1$ dimensional. Our desired conclusion will follow if we show that $H$ is exactly $m+1$ dimensional.

If $\operatorname{dim} H>m+1$, choose $s$ in $B$ such that $s \perp \bigvee\{P, v\}, s \neq 0$. This is possible, for $B$ being dense in $H$, choose any $s_{1}$ in $B$, not in $\mathrm{V}\{P, v\}$, and write $s_{1}=s_{2}+s$ with $s_{2}$ in $\bigvee\{P, v\}, s \perp \vee\{P, v\}$.

Since $s$ and $v \in B$, hence are bounded, there is a positive $c$ such that $1 \pm c s v>0$ on $X$, and since $|v(x)|=1$ a.e. $[G]$, we have $\int|v \pm c s| d G$ $=\int|1 \pm c s v| d G=\int[1 \pm c s v] d G=\int d G=1$, because $s \perp v$. We now define $F_{3}$ and $F_{4}$ in $B^{*}$ by $d F_{3}=(v+c s) d G$, and $d F_{4}=(v-c s) d G$. Then $F_{3}$ and $F_{4} \in N \cap S$, clearly. Also, $F_{3}$ and $F_{4} \in C$ because, for example, 
$\int e_{0} d F_{3}=\left\|e_{0}\right\| \int v(v+c s) d G=\left\|e_{0}\right\| \int v^{2} d G=\left\|e_{0}\right\|$. Finally, $F_{0}=\left(F_{3}+F_{4}\right) / 2$, and we have reached our contradiction, because $F_{0}$ is an extreme point of $C$.

We are now ready to prove our theorem.

Suppose, then, that $p_{0}$ satisfies $\left\|f-p_{0}\right\| \leqq\|f-p\|$ for every $p$ in $P$; $p_{0} \in P$. Let $F_{0}$ be an extreme point of $C$. Then $\Lambda\left(F_{0}\right)$ contains a finite number $m+1$ points, $m \leqq n$. For the integer $r$ of our theorem, we take $r=m$, and take $x_{0}, \cdots, x_{r}$ to be the points of $\Lambda\left(F_{0}\right)$. The $n \times(r+1)$ matrix $\left\{g_{i}\left(x_{j}\right)\right\}, i=1,2, \cdots, n ; j=0,1, \cdots, r$, must have rank $r$, since its row space is isomorphic to $P_{0}$, of dimension $m=r$. By renumbering the $g_{i}$, we may suppose that the first $r$ rows of our matrix are independent.

Now our measure $F_{0}$ is of the form $\int h d F_{0}=\sum_{j=0}^{r} h\left(x_{j}\right) m_{j}$ for every $h$ in $B$. Since $F_{0} \in N$, the $m_{j}$ must satisfy

$$
\left(\begin{array}{ccc}
g_{1}\left(x_{0}\right) & \cdots & g_{1}\left(x_{r}\right) \\
\vdots & & \vdots \\
g_{r}\left(x_{0}\right) & \cdots & g_{r}\left(x_{r}\right)
\end{array}\right)\left(\begin{array}{c}
m_{0} \\
\cdot \\
\dot{m_{r}}
\end{array}\right)=0 .
$$

The coefficient matrix having rank $r$ implies that the $m_{j}$ are given uniquely apart from a scalar factor by the cofactors of $a_{j}$ in the matrix

$$
T=\left(\begin{array}{ccc}
a_{0} & \cdots & a_{r} \\
g_{1}\left(x_{0}\right) & \cdots & g_{1}\left(x_{r}\right) \\
\vdots & & \vdots \\
g_{r}\left(x_{0}\right) & \cdots & g_{r}\left(x_{r}\right)
\end{array}\right),
$$

and the condition $\left\|F_{0}\right\|=1$, or $\sum_{0}^{r}\left|m_{j}\right|=1$, now fixes the $m_{j}$ apart from a factor \pm 1 . No $m_{j}$ can be zero here, or else $\Lambda\left(F_{0}\right)$ would contain fewer than $r+1$ points. Finally, the equation $\left\|e_{0}\right\|=\int e_{0} d F_{0}$ $=\sum_{0}^{r} e_{0}\left(x_{j}\right) m_{j}$ together with $\sum_{0}^{r}\left|m_{j}\right|=1$ imply that sgn $m_{j}$ $=\operatorname{sgn} e_{0}\left(x_{j}\right), j=0, \cdots, r$, and if we set $a_{j}$ equal to the sgn of its cofactor in the matrix $T$, we have $a_{j}=\operatorname{sgn} m_{j}, j=0, \cdots, r$, or else $a_{j}=-\operatorname{sgn} m_{j}, j=0, \cdots, r$, and we conclude that $e_{0}\left(x_{j}\right)=u a_{j}\left\|e_{0}\right\|$, $u=1$ or $u=-1$, and our theorem is half proved.

Conversely, if for some $r \leqq n$, the $n \times(r+1)$ matrix $\left\{g_{i}\left(x_{j}\right)\right\}$, $i=1, \cdots, n ; j=0,1, \cdots, r$ has rank $r$ for some $r+1$ distinct points $x_{0}, \cdots, x_{r}$, and if $a_{j}$ is the sgn of its cofactor in the matrix $T$ above, supposing the first $r$ rows of our $n \times(r+1)$ matrix are independent, and if, furthermore, we have $e_{0}\left(x_{j}\right)=a_{j} u\left\|e_{0}\right\|$ for all $j$ such that $a_{j} \neq 0, j=0, \cdots, r$, with $u= \pm 1$, then we assert $\left\|f-p_{0}\right\|$ $\leqq\|f-p\|$ for all $p$ in $P$. 
In fact, if $\|f-q\|<\left\|f-p_{0}\right\|=\left\|e_{0}\right\|$ for some $q$ in $P$, then for every $F$ in $N \cap S$, we have $\int f d F=\int(f-q) d F \leqq\|f-q\|<\left\|e_{0}\right\|$. On the other hand, we can construct an $F_{0}$ in $N \cap S$ for which $\int f d F_{0}=\left\|e_{0}\right\|$. Indeed, we need only solve the system

$$
\left(\begin{array}{ccc}
g_{1}\left(x_{0}\right) & \cdots & g_{1}\left(x_{r}\right) \\
\cdot & & \vdots \\
g_{r}\left(x_{0}\right) & \cdots & g_{r}\left(x_{r}\right)
\end{array}\right)\left(\begin{array}{c}
m_{0} \\
: \\
\dot{m_{r}}
\end{array}\right)=0
$$

with $\sum_{0}^{r}\left|m_{j}\right|=1$. The coefficient matrix has rank $r$, so the solution $m_{j}$ is given, apart from a scalar, by the cofactor of $a_{j}$ in $T$, above, and not all the $m_{j}$ are zero. Since $\sum_{0}^{r}\left|m_{j}\right|=1$, the measure $F_{0}$, given by $\int h d F_{0}=\sum_{0}^{r} h\left(x_{j}\right) m_{j}$, is in $N \cap S$. We now have

$$
\int f d F_{0}=\int\left(f-p_{0}\right) d F_{0}=\int e_{0} d F_{0}=\sum_{j=0}^{r} e_{0}\left(x_{j}\right) m_{j}=u\left\|e_{0}\right\| \sum_{0}^{r} a_{j} m_{j},
$$

and since $\sum_{0}^{r} a_{j} m_{j}= \pm 1$, we have $\int f d F_{0}= \pm\left\|e_{0}\right\|$; if $\int f d F_{0}=-\left\|e_{0}\right\|$. take $-F_{0}$ instead of $F_{0}$. The proof of the theorem is now complete.

Bureau of Ships, Washington, D. C. 